\title{
A General Minimax Disparity OWA Operator Optimization Problem
}

\section{Dug Hun Hong}

Department of Mathematics, Myongji University, Yongin, Korea

\section{ljfis}

Received: Jun. 26, 2018

Revised : Sep. 21, 2018

Accepted: Dec. 19, 2018

Correspondence to: Dug Hun Hong

(dhhong@mju.ac.kr)

(CThe Korean Institute of Intelligent Systems

(C)This is an Open Access article distributed under the terms of the Creative Commons Attribution Non-Commercial License (http://creativecommons.org/licenses/ by-nc/3.0// which permits unrestricted noncommercial use, distribution, and reproduction in any medium, provided the original work is properly cited.

\begin{abstract}
This paper provides an improvement of the general minimax disparity ordered weighted averaging (OWA) operator problem under more generalized assumption of the OWA operator. The work builds upon previous work regarding a general model of OWA aggregation of a given "orness" level.
\end{abstract}

Keywords: Fuzzy sets, OWA operator, Minimax disparity

\section{Introduction}

Yager [1] introduced a new aggregation technique based on the ordered weighted averaging (OWA) as a method for aggregating multiple units within max and min operators. Previous studies have suggested a number of approaches for obtaining the associated weights in different areas such as decision making, neural networks, expert systems, date mining, approximate reasoning, fuzzy system and control [1-11]. An OWA operator of dimension $n$ is a mapping $F: R^{n} \rightarrow R$ that has an associated weighting vector $W=\left(w_{1}, \cdots, w_{n}\right)$ of having the properties $w_{1}+\cdots+w_{n}=1, \quad 0 \leq w_{i} \leq 1, i=1, \cdots, n$, and such that

$$
F\left(a_{1}, \cdots, a_{n}\right)=\sum_{i=1}^{n} w_{i} b_{i},
$$

where $b_{j}$ is the $j$ th largest element of the collection of the aggregated objects $\left\{a_{1}, \cdots, a_{n}\right\}$. In [1], the author introduced a measure of "orness" associated with the weighting vector $W$ of an OWA operator, defined as

$$
\operatorname{orness}(W)=\sum_{i=1}^{n} \frac{n-i}{n-1} w_{i},
$$

and characterizes the degree to which the aggregation is like an or operation. Here, "orness" is interpreted as the mode of decision-making circumstances by conferring a meaning to the weights used in the process aggregation. An important issue in the theory of OWA operators is the determination of the associated weights, such as the methodology developed by O'Hagan [9]. In this methodology, the vector of the OWA weights can be calculated for a previously defined level of "orness".

Wang and Parkan [11] first proposed a minimax disparity OWA operator optimization problem:

$$
\text { Minimize } \quad \operatorname{Max}_{i \in\{1, \cdots, n-1\}}\left|w_{i}-w_{i+1}\right|
$$


subject to $\operatorname{orness}(W)=\sum_{i=1}^{n} \frac{n-i}{n-1} w_{i}=\alpha, \quad 0 \leq \alpha \leq 1$,

$$
w_{1}+\cdots+w_{n}=1, \quad 0 \leq w_{i}, \quad i=1, \cdots, n \text {. }
$$

The minimax disparity approach obtains OWA operator weights based on the minimization of the maximum difference between any two adjacent weights. The new approach was determined to be simpler than existing approaches due the requirement of only the formulation and solution of a linear programming model compared to other methods that require complex non-linear equations.

Recently, Liu [8, Theorem 6] considered the general minimax disparity OWA operator optimization problem:

\section{* The general minimax disparity OWA operator problem}

$$
\begin{aligned}
& \text { Minimize } \operatorname{Max}_{i \in\{1, \cdots, n-1\}}\left|F^{\prime}\left(w_{i}\right)-F^{\prime}\left(w_{i+1}\right)\right| \\
& \text { subject to } \operatorname{orness}(W)=\sum_{i=1}^{n} \frac{n-i}{n-1} w_{i}=\alpha, \\
& 0 \leq \alpha \leq 1 \\
& w_{1}+\cdots+w_{n}=1 \\
& w_{i} \geq 0, i=1, \cdots, n .
\end{aligned}
$$

where $F$ is a strictly convex function on $[0, \infty)$, and it is at least two order differentiable.

In this model, a consistent OWA operator is obtained allowing the aggregation value of the operator to monotonically changed with the given "orness" level. Further, a general regular increasing monotone quantifier determination was proposed and solved using the optimal control technique. The work of Liu demonstrated a key optimization model to incorporate background knowledge or required characteristics of aggregation problems.

In this paper, we give an improved version of the result for the problem (1) with assuming continuous first differentiability of $F$ instead of the second differentiability of $F$.

\section{General Minimax Disparity OWA Operator Optimization Problem}

It is noted that $F$ is strictly convex if and only if $F^{\prime}$ is strictly increasing. If $F^{\prime}(x)$ is strictly increasing, then there are two possible cases:

$$
F^{\prime}\left(0^{+}\right)=-\infty, \quad F^{\prime}\left(0^{+}\right)<\infty
$$

where $\lim _{x \rightarrow 0^{+}}=F^{\prime}\left(0^{+}\right)$. In case of $F^{\prime}\left(0^{+}\right)<\infty$, we define $F^{\prime}\left(0^{+}\right)=F^{\prime}(0)$.

Theorem 2.1. Assume that $F$ is strictly convex and $F^{\prime}(x)$ is continuous. The optimal solution for problem (1) with given orness level $0<\alpha<1$ is the weighting function

$$
w_{i}^{*}=\max \left\{\left(F^{\prime}\right)^{-1}\left(a^{*} i+b^{*}\right), 0\right\},
$$

where $a^{*}, b^{*}$ are determined by the constraints:

$$
\left\{\begin{array}{l}
\sum_{i \in H} \frac{n-i}{n-1}\left(F^{\prime}\right)^{-1}\left(a^{*} i+b^{*}\right)=\alpha, \\
\sum_{i \in H}\left(F^{\prime}\right)^{-1}\left(a^{*} i+b^{*}\right)=1,
\end{array}\right.
$$

and $H=\left\{i \mid\left(F^{\prime}\right)^{-1}\left(a^{*} i+b^{*}\right)>0\right\}$.

Proof. By the dual property [Theorem 5, 7] of OWA operator to generate OWA operator weights, it suffices only to prove the case of $1 / 2 \leq \alpha \leq 1$. We clearly have $W^{*}=\left(\frac{1}{n}, \frac{1}{n}, \cdots, \frac{1}{n}\right)$ for $\alpha=1 / 2$ and $W^{*}=(1,0, \cdots, 0)$ for $\alpha=1$. It is easy to check that $a^{*}<0$ for the case of $1 / 2 \leq \alpha \leq 1$.. Indeed, we show that $W^{*}=\left(w_{1}^{*}, \cdots, w_{s}^{*}, 0, \cdots, 0\right), 2 \leq s \leq n$ is the unique optimum solution of the constrained optimization problem (1) that satisfies the orness constraint.

Let $w_{i}^{*}=\max \left\{\left(F^{\prime}\right)^{-1}\left(a^{*} i+b^{*}\right), 0\right\}$ such that

$$
\begin{aligned}
& \sum i w_{i}^{*}=n-(n-1) \alpha\left(\Leftrightarrow \sum_{i=1}^{n} \frac{n-i}{n-1} w_{i}^{*}=\alpha\right), \\
& \sum w_{i}^{*}=1 .
\end{aligned}
$$

Then we clearly have $F^{\prime}\left(w_{i}^{*}\right)=a^{*} i+b^{*}, i \in H$. If $F^{\prime}\left(0^{+}\right)=$ $-\infty$, then $\left(F^{\prime}\right)^{-1}(x)>0$ and $H=\{1,2, \cdots, n\}$. Hence

$$
\operatorname{Max}_{i \in\{1, \cdots, n-1\}}\left|F^{\prime}\left(w_{i}^{*}\right)-F^{\prime}\left(w_{i+1}^{*}\right)\right|=\left|a^{*}\right| .
$$

If $F^{\prime}\left(0^{+}\right)<\infty$, then $F^{\prime}\left(w_{i}^{*}\right)=\max \left\{a^{*} i+b^{*}, F^{\prime}(0)\right\}$. If $w_{i}^{*}=\left(F^{\prime}\right)^{-1}\left(a^{*} i+b^{*}\right)>0$ and $w_{i+1}^{*}=0$, then $\left(F^{\prime}\right)^{-1}\left(a^{*}(i+\right.$ $\left.1)+b^{*}\right) \leq 0$, and hence $a^{*}(i+1)+b^{*} \leq F^{\prime}(0)$. Hence

$$
\operatorname{Max}_{i \in\{1, \cdots, n-1\}}\left|F^{\prime}\left(w_{i}^{*}\right)-F^{\prime}\left(w_{i+1}^{*}\right)\right|=\left|a^{*}\right| .
$$

Now, let $w_{i}, i=1, \cdots, n$ satisfy

$$
\begin{aligned}
& \sum_{i=1}^{n} i w_{i}=n-(n-1) \alpha, \\
& \sum_{i=1}^{n} w_{i}=1, \quad 0 \leq w_{i}, \quad i=1, \cdots, n .
\end{aligned}
$$


Then we can prove that

$$
\operatorname{Max}_{i \in\{1, \cdots, n-1\}}\left|F^{\prime}\left(w_{i}\right)-F^{\prime}\left(w_{i+1}\right)\right| \geq\left|a^{*}\right|,
$$

which shows that $W^{*}$ is an optimal solution of the constrained optimization problem (1). To prove (4), we assume the contrary, that is,

$$
\operatorname{Max}_{i \in\{1, \cdots, n-1\}}\left|F^{\prime}\left(w_{i}\right)-F^{\prime}\left(w_{i+1}\right)\right|<\left|a^{*}\right| .
$$

Then

$$
w_{1}<w_{1}^{*}
$$

If not, then $w_{1} \geq w_{1}^{*}$ and hence $F^{\prime}\left(w_{1}\right) \geq F^{\prime}\left(w_{1}^{*}\right)=a^{*}+b^{*}$. By (5), we have

$$
\begin{aligned}
F^{\prime}\left(w_{2}\right)>F^{\prime}\left(w_{1}\right)-\left|a^{*}\right| & \geq F^{\prime}\left(w_{1}^{*}\right)-\left|a^{*}\right| \\
& =F^{\prime}\left(w_{1}^{*}\right)+a^{*}=F^{\prime}\left(w_{2}^{*}\right) .
\end{aligned}
$$

Similarly, by induction, we have that $F^{\prime}\left(w_{i}\right)>F^{\prime}\left(w_{i}^{*}\right)$ for all $i=3, \cdots, s$. Then we have that $w_{i}>w_{i}^{*}$ for all $i=1, \cdots, s$ and $w_{i} \geq w_{i}^{*}=0$ is trivial for $i=s+1, \cdots, n$ hence

$$
\sum_{i=1}^{n} w_{i}>\sum_{i=1}^{n} w_{i}^{*}=1,
$$

which is contradictory to (3). It follows that $w_{1}<w_{1}^{*}$.

Here, we need a lemma.

Lemma 2.2. Let $w_{i}, i=1, \cdots, n$ satisfy

$$
\begin{aligned}
& \sum_{i=1}^{n} i w_{i}=n-(n-1) \alpha, \\
& \sum_{i=1}^{n} w_{i}=1, \quad 0 \leq w_{i}, \quad i=1, \cdots, n .
\end{aligned}
$$

and suppose that

$$
\operatorname{Max}_{i \in\{1, \cdots, n-1\}}\left|F^{\prime}\left(w_{i}\right)-F^{\prime}\left(w_{i+1}\right)\right| \leq\left|a^{*}\right| .
$$

Then $w_{1} \geq w_{1}^{*}$.

Proof. If not, then $w_{1}<w_{1}^{*}$. Since $\sum_{i=1}^{n} w_{i}=\sum_{i=1}^{n} w_{i}^{*}=1$, there exists $i_{0} \leq s$ such that $w_{i}<w_{i}^{*}$ for $i=1, \cdots, i_{0}-1$ and $w_{i_{0}} \geq w_{i_{0}}^{*}$. Then by (8), we have

$$
\begin{aligned}
F^{\prime}\left(w_{i_{0}+1}\right) & \geq F^{\prime}\left(w_{i_{0}}\right)-\left|a^{*}\right| \\
& \geq F^{\prime}\left(w_{i_{0}}^{*}\right)-\left|a^{*}\right| \\
& =F^{\prime}\left(w_{i_{0}}^{*}\right)+a^{*}
\end{aligned}
$$

$$
=F^{\prime}\left(w_{i_{0}+1}^{*}\right)
$$

and hence $w_{i_{0}+1} \geq w_{i_{0}+1}^{*}$. Similarly, by induction, we have $w_{i} \geq w_{i}^{*}$ for all $i=i_{0}+2, \cdots, s$ and $w_{i} \geq w_{i}^{*}$ is trivial for $i=s+1, \cdots, n$. Then we have that

$$
\begin{aligned}
& \sum_{i=1}^{n} i w_{i}-\sum_{i=1}^{n} i w_{i}^{*} \\
& =\sum_{i=1}^{n} i\left(w_{i}-w_{i}^{*}\right) \\
& =\sum_{i<i_{0}} i\left(w_{i}-w_{i}^{*}\right)+\sum_{i \geq i_{0}} i\left(w_{i}-w_{i}^{*}\right) \\
& \geq \sum_{i<i_{0}} i\left(w_{i}-w_{i}^{*}\right)+i_{0} \sum_{i \geq i_{0}}\left(w_{i}-w_{i}^{*}\right) \\
& =\sum_{i<i_{0}} i\left(w_{i}-w_{i}^{*}\right)-i_{0} \sum_{i<i_{0}}\left(w_{i}-w_{i}^{*}\right) \\
& =\sum_{i<i_{0}}\left(i-i_{0}\right)\left(w_{i}-w_{i}^{*}\right) \\
& >0
\end{aligned}
$$

where the second equality comes from the fact that $\sum_{i<i_{0}}\left(w_{i}-\right.$ $\left.w_{i}^{*}\right)=-\sum_{i \geq i_{0}}\left(w_{i}-w_{i}^{*}\right)$. This means that $\sum_{i=1}^{n} i w_{i}>$ $\sum_{i=1}^{n} i w_{i}^{*}=n-(n-1) \alpha$, which is contradictory to (7).

Back to the proof of Theorem 2.1. We also have $w_{1} \geq w_{1}^{*}$ by Lemma 2.2, which is contradictory to (6). It follows that

$$
\operatorname{Max}_{i \in\{1, \cdots, n-1\}}\left|F^{\prime}\left(w_{i}\right)-F^{\prime}\left(w_{i+1}\right)\right| \geq\left|a^{*}\right|,
$$

and hence $W^{*}=\left(w_{1}^{*}, \cdots, w_{s}^{*}, 0, \cdots, 0\right), 2 \leq s \leq n$ is a optimum solution of the constrained optimization problem (1). Now, we show that $W^{*}$ is the unique optimum solution of the constrained optimization problem (1). Let $w_{i}, i=1, \cdots, n$ satisfy

$$
\begin{aligned}
& \sum_{i=1}^{n} i w_{i}=n-(n-1) \alpha \\
& \sum_{i=1}^{n} w_{i}=1,0 \leq w_{i}, i=1, \cdots, n .
\end{aligned}
$$

and suppose that

$$
\operatorname{Max}_{i \in\{1, \cdots, n-1\}}\left|F^{\prime}\left(w_{i}\right)-F^{\prime}\left(w_{i+1}\right)\right|=\left|a^{*}\right| .
$$

By Lemma 2.2 , we have $w_{1} \geq w_{1}^{*}$. Then by (9), we have

$$
F^{\prime}\left(w_{2}\right) \geq F^{\prime}\left(w_{1}\right)-\left|a^{*}\right|
$$




$$
\begin{aligned}
& \geq F^{\prime}\left(w_{1}^{*}\right)-\left|a^{*}\right| \\
& =F^{\prime}\left(w_{1}^{*}\right)+a^{*} \\
& =F^{\prime}\left(w_{2}^{*}\right)
\end{aligned}
$$

and hence $w_{2} \geq w_{2}^{*}$. Similarly, by induction, we have that $w_{i} \geq w_{i}^{*}$ for $i=3, \cdots, s$, and $w_{i} \geq w_{i}^{*}$ is trivial for $i=s+1$, $\cdots, n$. Now, since $w_{i}-w_{i}^{*} \geq 0, i=1,2, \cdots, n$ and $0=$ $\sum_{i=1}^{n}\left(w_{i}-w_{i}^{*}\right)$, we have that $w_{i}=w_{i}^{*}$ for all $i=1, \cdots, n$, which completes the proof.

\section{Numerical Example}

We consider a OWA operator $F$ which is not differentiable to at least the second order but $F^{\prime}$ is absolutely continuous and find a optimal solution of two OWA problem.

Let a OWA operator $F$ be

$$
F(x)= \begin{cases}\frac{x^{2}}{2}, & \text { if } 0 \leq x<\frac{1}{2}, \\ x^{2}-\frac{1}{2} x+\frac{1}{8}, & \text { if } \frac{1}{2} \leq x \leq 1 .\end{cases}
$$

Then

$$
F^{\prime}(x)= \begin{cases}x, & \text { if } 0 \leq x<\frac{1}{2}, \\ 2 x-\frac{1}{2}, & \text { if } \frac{1}{2} \leq x \leq 1 .\end{cases}
$$

Hence $F(x)$ is strictly convex and $F^{\prime}(x)$ is continuous, but $F(x)$ is not second order differentiable. Then we cannot apply the result of Liu [8, Theorem 6] but our result, Theorem 2.1. By the dual property [8, Theorem 5] of OWA to generate OWA operate weights, it suffices only to prove the case $\frac{1}{2} \leq \alpha \leq 1$. Then the OWA operator vector has the form

$$
W^{*}=\left(w_{1}^{*}, w_{2}^{*}, \cdots, w_{m}^{*}, 0,0, \cdots, 0\right),
$$

where $m \in H, m-1 \notin H$ and

$$
w_{i}^{*}= \begin{cases}\left(F^{\prime}\right)^{-1}\left(a^{*} i+b^{*}\right), & 1 \leq i \leq m, \\ 0, & m<i \leq n .\end{cases}
$$

Since $\left(F^{\prime}\right)^{-1}(x)>\frac{1}{2}$ for $x \in\left(\frac{1}{2}, 1\right]$, there exist only two cases.

Case 1) $F^{\prime}\left(w_{1}^{*}\right)=a^{*}+b^{*}>\frac{1}{2}$

If $a^{*}+b^{*}>\frac{1}{2}$ then

$$
w_{i}^{*}=\left(F^{\prime}\right)^{-1}\left(a^{*} i+b^{*}\right)
$$

$$
= \begin{cases}\frac{1}{2} a^{*}+\frac{1}{2} b^{*}+\frac{1}{4}, & i=1, \\ a^{*} i+b^{*}, & 2 \leq i \leq m,\end{cases}
$$

where $m \in H$ and $m+1 \notin H$. From

$$
\left\{\begin{array}{l}
\sum_{i=1}^{n} \frac{n-i}{n-1} w_{i}^{*}=\alpha, \\
\sum_{i=1}^{n} w_{i}=1,
\end{array}\right.
$$

we can get

$$
\begin{aligned}
a^{*}= & -\frac{3\{3 m(m-1)-4(1-\alpha)(n-1)(2 m-1)\}}{2 m(m-1)\left(m^{2}-m+1\right)}, \\
b^{*}= & \frac{3 m(m-1)(m+1)}{m(m-1)\left(m^{2}-m+1\right)} \\
& -\frac{6(1-\alpha)(n-1)\left(m^{2}+m-1\right)}{m(m-1)\left(m^{2}-m+1\right)} .
\end{aligned}
$$

Let $\alpha_{1}$ and $\alpha_{2}$ be solutions of $a^{*}+b^{*}=\frac{1}{2}$ and $w_{m}^{*}=a^{*} m+$ $b^{*}=0$, respectively. Then

$$
\begin{aligned}
& \alpha_{1}=\frac{m^{2}-7 m-8+12 n}{12(n-1)}, \\
& \alpha_{2}=-\frac{m^{2}+4 m-1-4 m n}{4 m(n-1)} .
\end{aligned}
$$

Since $a^{*}+b^{*}$ is increasing and $w_{m}^{*}$ is decreasing as $\alpha$ is increasing, $a^{*}+b^{*}>\frac{1}{2}$ and $w_{m}^{*}>0$ implies $\alpha_{1}<\alpha<\alpha_{2}$. Therefore

$$
\begin{aligned}
& \alpha_{2}-\alpha_{1} \\
& =-\frac{m^{2}+4 m-1-4 m n}{4 m(n-1)}-\frac{m^{2}-7 m-8+12 n}{12(n-1)} \\
& =-\frac{(m-4)\left(m^{2}-m+1\right)}{4(n-1)(m-1)}>0 .
\end{aligned}
$$

This implies that $m<4$.

If $m=3$ then,

$$
\begin{aligned}
& \alpha_{2}=\frac{3 n-5}{3(n-1)}, \\
& a^{*}=-\frac{9-10(1-\alpha)(n-1)}{14}, \\
& b^{*}=\frac{12-11(1-\alpha)(n-1)}{7},
\end{aligned}
$$

and if $m=2$, then

$$
\alpha_{2}=\frac{8 n-11}{8(n-1)},
$$




$$
\begin{aligned}
& a^{*}=-\frac{3-6(1-\alpha)(n-1)}{2}, \\
& b^{*}=3-5(1-\alpha)(n-1) .
\end{aligned}
$$

So

$$
w_{i}^{*}= \begin{cases}\frac{11-6(1-\alpha)(n-1)}{14}, & i=1, \\ -\frac{9-10(1-\alpha)(n-1)}{14} i & \\ +\frac{12-11(1-\alpha)(n-1)}{7}, & i=2,3, \\ 0, & 3<i \leq n,\end{cases}
$$

for $\alpha \in\left(\frac{3 n-5}{3(n-1)}, \frac{8 n-11}{8(n-1)}\right]$, and

$$
w_{i}^{*}= \begin{cases}1-(1-\alpha)(n-1), & i=1, \\ (1-\alpha)(n-1), & i=2, \\ 0, & 2<i \leq n,\end{cases}
$$

for $\alpha \in\left(\frac{8 n-11}{8(n-1)}, 1\right]$.

Case 2) $F^{\prime}\left(w_{1}^{*}\right)=a^{*}+b^{*} \leq \frac{1}{2}$

If $a^{*}+b^{*} \leq \frac{1}{2}$ then $m \geq 4$ and $\left(F^{\prime}\right)^{-1}\left(a^{*} i+b^{*}\right)=a^{*} i+b^{*}$ for all $1 \leq i \leq m$. From

$$
\left\{\begin{array}{l}
\sum_{i=1}^{n} \frac{n-i}{n-1} w_{i}^{*}=\alpha \\
\sum_{i=1}^{n} w_{i}=1
\end{array}\right.
$$

we can get

$$
\begin{aligned}
& a^{*}=-\frac{6\{m-1-2(1-\alpha)(n-1)\}}{m(m-1)(m+1)}, \\
& b^{*}=\frac{2\{2(m-1)-3(1-\alpha)(n-1)\}}{m(m-1)} .
\end{aligned}
$$

Hence $w_{m+1}^{*} \leq 0<w_{m}^{*}$ implies

$$
1+3(1-\alpha)(n-1)<m \leq 2+3(1-\alpha)(n-1),
$$

and so

$$
\begin{aligned}
w_{i}^{*}= & -\frac{6\{m-1-2(1-\alpha)(n-1)\}}{m(m-1)(m+1)} i \\
& +\frac{2\{2(m-1)-3(1-\alpha)(n-1)\}}{m(m-1)}
\end{aligned}
$$

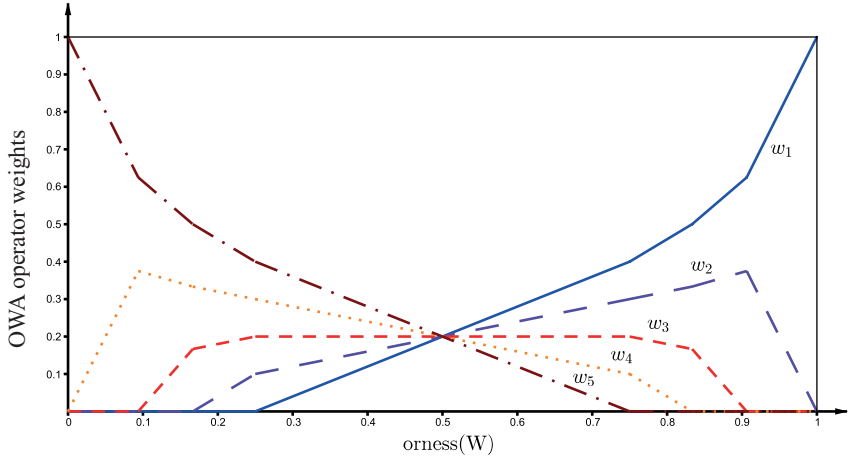

Figure 1. The OWA operator weihts for $F(x)$ with $n=5$.

for $1 \leq i \leq m$ and $w_{i}^{*}=0$ for $m<i \leq n$, where

$$
m=\min \{n,[2+3(1-\alpha)(n-1)]\},
$$

and $\frac{3 n-m-2}{3(n-1)}<\alpha \leq \frac{3 n-m-1}{3(n-1)}$.

For example, if $n=5$, then we have for $\alpha \in\left(\frac{29}{32}, 1\right], m=2$

$$
w_{i}^{*}= \begin{cases}4 \alpha-3, & i=1, \\ 4-4 \alpha, & i=2, \\ 0, & 2<i \leq 5,\end{cases}
$$

for $\alpha \in\left(\frac{5}{6}, \frac{29}{32}\right], m=3$

$$
w_{i}^{*}= \begin{cases}\frac{24 \alpha-13}{14}, & i=1, \\ -\frac{40 \alpha-31}{14} i+\frac{44 \alpha-32}{7}, & i=2,3, \\ 0, & 3<i \leq n,\end{cases}
$$

for $\alpha \in\left(\frac{3}{4}, \frac{5}{6}\right], m=4$

$$
w_{i}^{*}= \begin{cases}-\frac{8 \alpha-5}{10} i+2 \alpha-1, & 1 \leq i \leq 4, \\ 0, & i=5\end{cases}
$$

and for $\alpha \in\left[\frac{1}{2}, \frac{3}{4}\right], m=5$

$$
w_{i}^{*}=-\frac{2 \alpha-1}{5} i+\frac{6 \alpha-2}{5}, \quad 1 \leq i \leq 5 .
$$

\section{Conclusion}

An improvement of the general minimax disparity OWA operator problem developed by Liu [8] is presented. A more generalized assumption of the OWA operator is utilized to build 
upon previous work regarding a general aggregation model for a given "orness" level.

\section{Acknowledgements}

This research was supported by Basic Science Research Program through the National Research Foundation of Korea (NRF) funded by the Ministry of Education (2017R1D1A1B03027869).

\section{References}

[1] R. R. Yager, "On ordered weighted averaging aggregation operators in multicriteria decisionmaking," IEEE Transactions on Systems, Man, and Cybernetics, vol. 18, no. 1, pp. 183-190, 1988. https://doi.org/10.1109/21.87068

[2] B. S. Ahn, "Preference relation approach for obtaining OWA operator weights," International Journal of Approximate Reasoning, vol. 47, no. 2, pp. 166-178, 2008. https://doi.org/10.1016/j.ijar.2007.04.001

[3] D. Filev and R. R. Yager, "Analytic properties of maximum entropy OWA operators," Information Sciences, vol. 85, no. 1-3, pp. 11-27, 1995. https://doi.org/10.1016/00200255(94)00109-O

[4] R. Fuller and P. Majlender, "An analytic approach for obtaining maximal entropy OWA operator weights," Fuzzy Sets and Systems, vol. 124, no. 1, pp. 53-57, 2001. https: //doi.org/10.1016/S0165-0114(01)00007-0

[5] R. Fuller and P. Majlender, "On obtaining minimal variability OWA operator weights," Fuzzy Sets and Systems, vol. 136, no. 2, pp. 203-215, 2003. https://doi.org/10.1016/ S0165-0114(02)00267-1

[6] D. H. Hong, "A note on the minimal variability OWA operator weights," International Journal of Uncertainty, Fuzziness and Knowledge-Based Systems, vol. 14, no. 6, pp. 747-752, 2006. https://doi.org/10.1142/ S0218488506004308
[7] D. H. Hong, "On proving the extended minimax disparity OWA problem," Fuzzy Sets and Systems, vol. 168, no. 1, pp. 35-46, 2011. https://doi.org/10.1016/j.fss.2010.08.008

[8] X. Liu, "A general model of parameterized OWA aggregation with given orness level," International Journal of Approximate Reasoning, vol. 48, no. 2, pp. 598-627, 2008. https://doi.org/10.1016/j.ijar.2007.11.003

[9] M. O'Hagan, "Aggregating template or rule antecedents in real-time expert systems with fuzzy set logic," in Proceedings of the 22nd Asilomar Conference on Signals, Systems and Computers, Pacific Grove, CA, 1988, pp. 681-689. https://doi.org/10.1109/ACSSC.1988.754637

[10] X. Sang and X. Liu, "An analytic approach to obtain the least square deviation OWA operator weights," Fuzzy Sets and Systems, vol. 240, pp. 103-116, 2014. https://doi.org/ 10.1016/j.fss.2013.08.007

[11] Y. M. Wang and C. Parkan, "A minimax disparity approach obtaining OWA operator weights," Information Sciences, vol. 175, no. 1-2, pp. 20-29, 2005. https://doi. org/10.1016/j.ins.2004.09.003

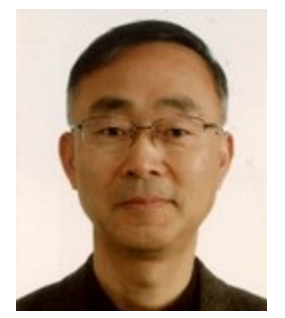

Dug Hun Hong received the B.S. and M.S. degrees in Mathematics from Kyungpook National university, Daegu Korea in 1981 and 1983, respectively. He received the M.S. and Ph.D. degrees from the University of Minnesota in 1988 and 1990, respectively. His research interests include probability theory and fuzzy theory with applications. E-mail: dhhong@mju.ac.kr 